\title{
Dense suspensions - solid-liquid interactions at the
} particle scale

\section{J.J. Derksen}

Chemical and Materials Engineering, University of Alberta,

Edmonton, Alberta,

T6G 2G6 Canada

E-mail: jos@ualberta.ca

\begin{abstract}
Flows of solid-liquid suspensions span a multi-dimensional parameter space, with coordinates such as the Stokes number, the solids volume fraction, the density ratio, and Reynolds numbers. We are interested in systems with appreciable inertia effects - i.e., non-zero Stokes and Reynolds numbers - having density ratios of the order of one (typical for solid-liquid systems) and solids volume fractions of at least 0.1 . Additional effects include strongly inhomogeneous solids distributions, non-Newtonian liquids, and sticky particles that tend to aggregate. This leads to a rich spectrum of interactions at the scale of individual particles. To reveal these we perform direct simulations of collections of a few thousand of particles carried by a liquid flow with resolution of the solid-liquid interfaces. For this we use the lattice-Boltzmann method supplemented with an immersed boundary approach.
\end{abstract}

Keywords: multiphase flow; suspensions; direct numerical simulation; LBM; lattice-Boltzmann method; immersed boundary method; mesoscopic modelling; particle-turbulence interaction; aggregation; collision modelling.

Reference to this paper is made as follows: Derksen, J.J. (2012) 'Dense suspensions - solid-liquid interactions at the particle scale', Progress in Computational Fluid Dynamics, Vol. 12, No. 2/3, pp.103-111.

Biographical notes: J.J. Derksen, PhD degree (1991) in Applied Physics from Eindhoven University of Technology (Netherlands). Research Engineer at the Dutch Aerospace Laboratory (NLR) 1991-1992. Assistant Professor of Applied Physics at Delft University of Technology (Netherlands) 1992-2007. Professor of Chemical Engineering at the University of Alberta (Canada) since 2007.

\section{Introduction}

Solid-liquid suspensions are abundant in natural and engineered systems. Our interest in large-scale industrial multiphase flows implies that we have systems with appreciable inertia effects, having density ratios of the order of one (solid-liquid) and high disperse phase loading (solids volume fractions of order 0.1). In such suspensions many assumptions that ease the life of the computational researcher do not hold, and direct simulations - including full resolution of the solid-liquid interfaces - are desired to reveal the relevant interactions at the scale of the particles. This necessarily limits the size of the systems that we are able to simulate; they typically contain up to a few thousand particles. In this paper, the scales related to particle size and multi-particle interaction will be termed particle-scales or (better) meso-scales, and our direct simulations are meso-scale simulations. Next to the ambition, to fully resolve meso-scale phenomena, we are faced with the issue as to how to incorporate insights gained at the meso-scale in macro-scale modelling approaches (meso-tomacro coupling). In the opposite direction (macro-to-meso), the meso-scale systems need to be agitated (energised) in a manner that realistically represents the energy input that in many practical processes comes from the macro-scale; think of agitation by impellers, jets, distributor plates, and pumps (generating overall pressure gradients).

Given the variety of multiple-scale interactions in industrial multiphase flow systems there is not a general methodology or framework for establishing the macro-meso coupling. In this paper, I will show examples of meso-scale simulations in the area of mostly turbulent liquid-solid suspensions, and show how their results could be incorporated in macroscopic flow and transport modelling.

The paper is organised in the following manner: First, we give a short overview of our computational methodology, which is largely based on the lattice-Boltzmann method LBM for solving the flow of the interstitial liquid. We then briefly describe methods for generating Homogeneous, Isotropic Turbulence (HIT) as a basic way to excite meso-scale systems. Subsequently applications will be discussed. They comprise turbulence-particle interaction and aggregation: flow-induced forces in agglomerates, and coagulation, breakage and structuring of aggregates in mostly turbulent flow fields. 


\section{Computational approach}

\subsection{Lattice-Boltzmann method}

The continuous phase (liquid) flow we solve with the LBM. For flows in complexly shaped domains and/or with moving boundaries, this method has proven its usefulness (see for e.g., the review article by Chen and Doolen, 1998). In the LBM, the computational domain is discretised into a number of lattice nodes residing on a uniform, cubic grid. Fluid parcels move from each node to its neighbours according to prescribed rules. It can be proven by means of a ChapmanEnskog expansion that - with the proper grid topology and collision rules - this system obeys, in the low Mach number limit, the incompressible Navier-Stokes equations (Chen and Doolen, 1998; Succi, 2001). The specific implementation used in our simulations has been described by Somers (1993), which is a variant of the widely used Lattice-BGK scheme to handle the collision integral (e.g., see Qian et al., 1992). We use the scheme due owing to Somers, as it manifests a more stable behaviour at low viscosities when compared to with standard LBGK.

\subsection{Liquid-solid and solid-solid coupling}

In the Lattice-Boltzmann flow field spherical (usually monosised), solid particles are suspended. The solid-liquid interfaces are fully resolved. The fluid flow and the motion of the spheres are coupled by demanding that at the surface of each sphere the fluid velocity matches the local velocity of its surface (that is the sum of the linear velocity $\mathbf{v}_{\mathrm{p}}$ and $\Omega_{p} \times\left(\mathbf{r}-\mathbf{r}_{\mathrm{p}}\right)$ with $\Omega_{p}$ the angular velocity of the sphere, $\mathbf{r}_{\mathrm{p}}$ the center position of the sphere, and $\mathbf{r}$ a point on its surface). In the forcing (aka immersed boundary) scheme that is applied here this is accomplished by imposing additional forces on the fluid at the surface of the solid sphere (which are then distributed to the lattice nodes in the vicinity of the particle surface). The details of the implementation can be found elsewhere (Goldstein et al,. 1993; Derksen and Van den Akker, 1999; Ten Cate et al., 2002). The collection of forces acting on the fluid at the sphere's surface and its interior is subsequently used to determine the hydrodynamic force and torque acting on the sphere (action $=-$ reaction) (Derksen and Sundaresan, 2007).

In our simulations, the radius of each spherical particle is specified and input radius refers to this radius scaled by the lattice spacing. In the LBM simulations, as the spherical particle is represented by forces that are confined to a cubic grid, the input radius does not reflect the actual radius of the particle. A calibration procedure to estimate the effective radius of this object (commonly referred to as the hydrodynamic radius) was introduced by Ladd (1994). We apply his scheme to estimate the hydrodynamic radius of the particles. The hydrodynamic radius is recognised as $a$ and is given in lattice units. In our work radii in the range $a=6-12$ are used. Typically the input radius turns out to be some half a lattice spacing or less smaller than the hydrodynamic radius.

In multiple-sphere systems when two spheres are in close proximity, with their separation being of the order of or less than the lattice spacing, the hydrodynamic interaction between them will not be accurately resolved by the lattice. Therefore, we explicitly impose lubrication forces on the spheres, in addition to the hydrodynamic forces stemming from the LBM. We use the procedure developed by Nguyen and Ladd (2002) to smoothly make the transition between resolved and unresolved hydrodynamic interactions.

In addition to the interactions via the liquid, spherical particles have direct interactions. In the first place the spheres collide. By default we use hard-sphere collisions according to a two-parameter model (Yamamoto et al., 2001) with a restitution coefficient $e$ and a friction coefficient $\mu$. In some situations where particle motion is constrained (such as with fibres built of strings of spheres) we - largely for reasons of computational efficiency - use soft-sphere collisions.

In order to study aggregation we can make the spheres sticky by giving them an attractive Square-Well Potential (SqWP) interaction (Smith et al., 1997): If the centrers of two approaching spheres come within a distance $2(\alpha+\delta)$ they trade potential energy for kinetic energy (by an amount $E_{\text {swp }}$ per sphere). Then, they then are within one another's SqWP and are considered attached. Two attached spheres can only separate if they are able to overcome the energy barrier imposed by the SqWP with their kinetic energy. The particle interaction potential is thus defined by two parameters: its depth $\left(E_{\text {swp }}\right)$ and its width $(\delta)$. Rather than working with $E_{\text {swp }}$ we will be working with the parameter $\Delta u$, which is the escape velocity of the SqWP. These parameters are related according to $E_{s w p}=\frac{1}{2} m_{p}(\Delta u)^{2}$ with $m_{p}$ the mass of the (monosized) primary spherical particles.

\subsection{Homogeneous isotropic turbulence}

In the A typical way to agitate our meso-scale systems is by generating turbulence in fully periodic, three-dimensional domains. Adding particles to the domains allows us to study the (two-way) coupling of solid and liquid motion. So far mainly HIT has been considered in our work. HIT is e.g., characterised by its root-mean-square velocity $u_{\text {rms }}$ and a Kolmogorov length scale $\eta_{K}=\left(v^{3} / \bar{\varepsilon}\right)^{1 / 4}$ with $\bar{\varepsilon}$ the volume and time averaged dissipation rate (which in steady state equals energy input) and $v$ the kinematic viscosity of the liquid. If solids are added, the relevant dimensionless numbers are then based on the radius of the spherical particles involved: and $\mathrm{Re}_{\text {rms }}=u_{\text {rms }} a / v$ and $a / \eta_{K}$.

We have been using two different strategies to make HIT. One is based on random forcing and was introduced by Alvelius (1999) in the context of spectral methods, later adapted for the lattice-Boltzmann method LBM by Ten Cate et al. (2006). This strategy has the advantages that the power input can be controlled accurately, and that it allows for more general forms of turbulence, including anisotropic turbulence with full control over the volume-averaged anisotropy tensor.

The second strategy is linear forcing, where turbulence is sustained by a force that is proportional to the local velocity (Rosales and Meneveau, 2005). This method has the elegance of simplicity and (as a result) computational efficiency at 
the cost of being less general than random forcing. Linear forcing does provide good control over the power input and thus (once equilibrium between power input and dissipation has been reached) over the Kolmogorov scales.

\section{Case studies}

\subsection{DNS of turbulently agitated solid-liquid suspensions}

With a view to applications in industrial crystallisation, Ten Cate et al. (2004) studied the motion of solid, spherical particles released in HIT. The conditions were such that $a / \eta_{K}$ was of the order of 10 , and $\mathrm{Re}_{\mathrm{rms}}$ roughly 50 . Attrition, i.e., breakage of crystals due owing to collisions is an important issue in crystallisation as it directly and indirectly influences the crystal size distribution. The direct influence is obvious; the indirect influence on the size distribution is a result of breakage fragments acting as a (secondary) sources of nucleation.

As is known from experimental (Elghobashi and Truesdell, 1993) as well as numerical work (Boivin et al., 1998), the presence of the particles affects the turbulence spectrum. Figure 1 shows spectra at different solids loadings. As evident from the spectra, and also from a direct look into our simulations, the spheres generate turbulence at scales comparable to with and smaller than the particle diameter. This effect is a pronounced function of the solids volume fraction: the more particles, the stronger the effect.

Our main interest was to quantify particle collisions, in terms of frequencies and intensities. In this respect it was revealing to study the Probability Density Function (PDF) of the time between two collisions of a particle (as given in Figure 2). For 'long times' this PDF is exponential indicating Poisson statistics; collisions after 'long' time intervals are uncorrelated events. The slopes in Figure 2 get steeper for denser systems, i.e., the average time between uncorrelated collisions gets shorter for denser systems. More interestingly, however, for 'short times' the PDF shows a peak towards zero time, indicating many collisions taking place shortly after one another. Closer inspection teaches that these are correlated

Figure 1 Energy spectra of two-phase simulations compared to the fluid-only spectrum. The wavenumber $\kappa$ is normalised with the particle size wavenumber $\kappa_{d}=\pi / a$

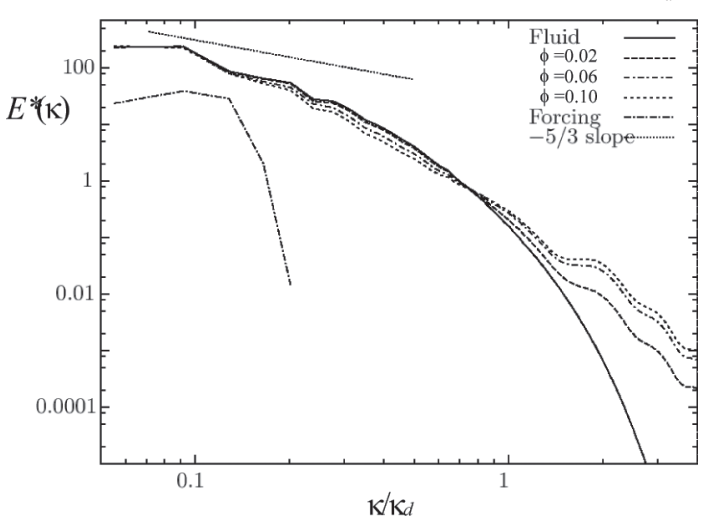

Figure 2 PDF of the time between two collisions for three solids volume fractions. The lines are linear fits of the tails of the distribution. The collision time has been made dimensionless with the Kolmogorov time scale $\tau$

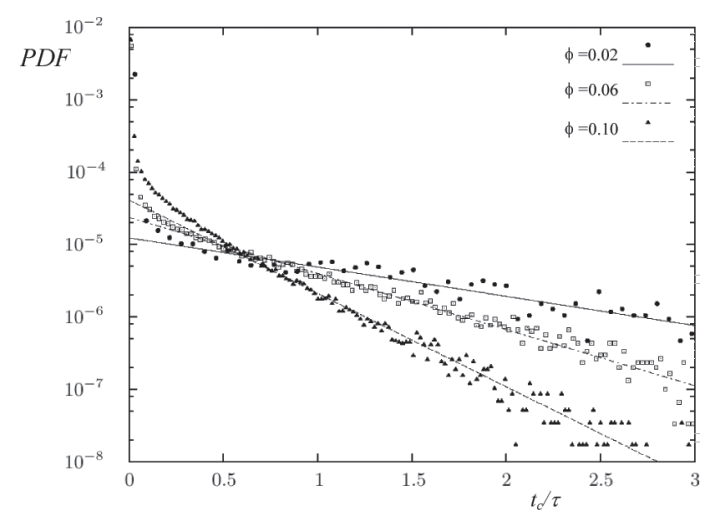

events: once turbulence has brought two (or more) particles in each other's vicinity they tend to cluster due owing to short range hydrodynamic interaction and undergo many (weak) collisions at short-time intervals. Lubrication forces play a prominent role in the lifetime of the clusters. Eventually, the particles in the cluster are separated when a strong enough (turbulent) eddy comes by.

In terms of macro-scale modelling, results related to collision statistics as a function of turbulence quantities and solids loading could be incorporated in (macro-scale) population balance modelling for predicting breakage and crystal size distributions.

\subsection{Flow-induced forces in agglomerates}

In many processes involving solid particle formation or solids handling, particles have a tendency to stick together. Sometimes agglomeration is a wanted phenomenon to effectively grow particles making separation easier. It also is a mechanism that potentially destroys a narrow particle size distribution, and as a result could deteriorate product quality. Much effort goes into preventing or promoting agglomeration, and much effort goes into repairing the harm agglomeration has done. Regardless of whether agglomeration is wanted or unwanted, it is relevant to assess the stability and the integrity of the bond holding the primary particles together. Agglomerates can break as a result of a variety of mechanisms, one of them being the flow of fluid surrounding the agglomerate: velocity gradients induce forces on and in agglomerates that could break them.

Understanding and modelling agglomerate breakage as a result of fluid flow is largely based on relatively simple concepts involving estimating shear rates and semi-empirical correlations for breakage statistics. As described in recent papers on the broader subject of population balance modelling of colloidal dispersions (Soos et al., 2006), the physical discription of breakage due owing to flow date back quite some time (Delichatsios and Probstein, 1976; Kusters, 1991), and is prone to refinement in terms of getting the (statistics of the) hydrodynamic environment of agglomerates right, 
and in terms of estimating the actual hydrodynamic forces in agglomerates immersed in complex flow.

As a starting point, we here assess the role of some of the non-ideal factors in the flow-induced forces in agglomerates. For this, we first have chosen to consider the virtually simplest agglomerate possible: two equally sized spheres (radius $a$ ), rigidly constrained together at their (single) point of contact, i.e., a sphere doublet. The two spheres are touching, they have zero separation.

We release a single doublet in HIT (now generated through linear forcing) and monitor the forces and torques at the point of contact needed to keep the two spheres attached. The time series are highly erratic (see the example in Fig. 3), with the fluctuation levels usually much higher than the averages. The positive average normal force in Figure 3 is the average (always tensile) centrifugal force. Running a number of simulations with $\left(a / \eta_{k}\right)$ as the main variable shows an interesting scaling of the force fluctuation levels. As $a / \eta_{K}$ increases, the flow around the agglomerate gets more inhomogeneous, which adds to the fluctuations, see Figure 4. More details and results can be found in a recent paper (Derksen, 2008).

Small-scale (micro) devices have also been used to perform experiments on aggregate breakage (Zaccone et al., 2009). In such devices the deformations in now laminar flow induce forces in the agglomerates. Here, we investigate these forces computationally.

The basic flow geometry for this is a square channel with width $H$. The flow in the channel is driven by a body force $f_{0}$ acting in the $x$ (=streamwise) direction mimicking a pressure gradient. At the four side walls a no-slip boundary condition

Figure 3 Time series of the flow-induced normal force at the point of contact of a sphere doublet. Time has been normalised with the Kolmogorov time scale $\tau$

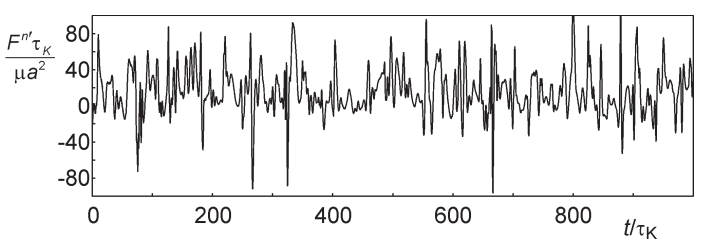

Figure 4 Root-mean-square $F^{n^{\prime}}$ normal force in the sphere doublet at various turbulence conditions, characterised by the ratio $a / \eta_{K}$ along with a trend line

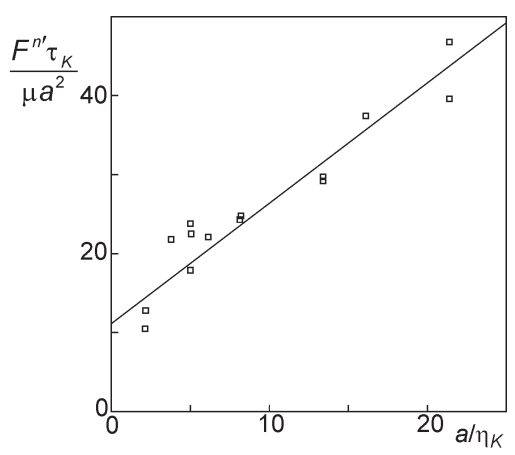

applies; the flow system is periodic in streamwise direction. A Reynolds number characterising the flow in the channel can be based on the wall shear velocity. Since the average wall shear stress relates to $f_{0}$ via an overall force balance we can write

$$
\operatorname{Re}_{w}=\frac{1}{2} \frac{H^{3 / 2} f_{0}^{1 / 2}}{\rho^{1 / 2} v} \text {. }
$$

In the liquid that fills the channel, agglomerates are released. They consist of equally sized spheres with radius $a$. Three types of agglomerates have been considered:

- two touching spheres forming a doublet

- $\quad$ three touching spheres (triplet) forming a triangle (two contact points per primary sphere)

- four touching spheres (quadruplet) forming a tetrahedron (three contact points per primary sphere).

The introduction of the agglomerates in the channel gives rise to three additional dimensionless numbers: an aspect ratio $a / H$, a density ratio $\rho_{\mathrm{s}} / \rho$, and a solids volume fraction $f$.

Figure 5 shows a typical flow situation with (in this case) quadruplets in a uniform square channel. The channel width $H$ is 10 times the primary sphere diameter.

In the triplets and quadruplets considered, primary spheres have more than one point of contact with the other spheres in the agglomerate. For these special cases the simulation procedure allows us to determine the forces and torques per contact point. As examples, time series of radial contact forces in quadruplets are shown in Figure 6. The smooth parts of the fluctuations shown are dueowing to motion of the

Figure 5 Cross sections through a uniform channel with quadruplets in terms of absolute liquid velocity contours. $\operatorname{Re}_{w}=2.6, \rho_{s} / \rho=2.5, \phi=0.062, a / H=0.05$ (see online version for colours)

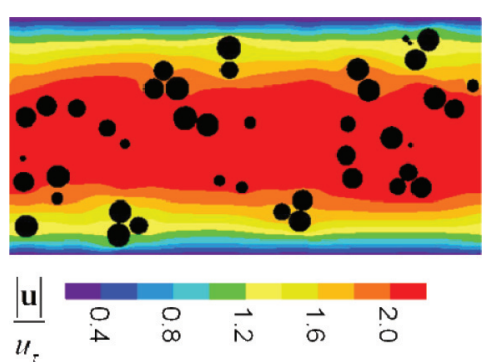

Figure 6 Time series of the dimensionless normal force in one sphere-sphere contact point in a quadruplet. Comparison for different slurry densities. Blue: solids volume fraction $9.3 \%$; red: $6.2 \%$; green: $3.1 \%$. Uniform channel (see online version for colours)

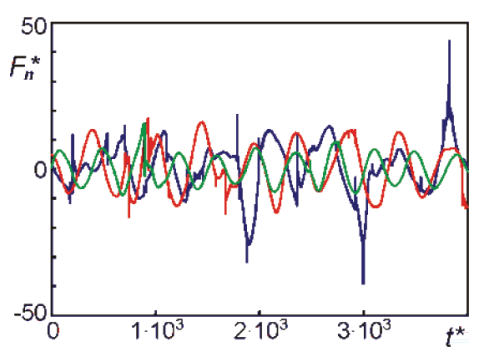


rotating agglomerate through the channel thereby sampling variations in the local liquid deformation rate. The spikes and discontinuities are dueowing to encounters with other agglomerates; the denser the suspension, the more encounters. One way of summarising the detailed information provided by the simulations is in the form of PDF's of contact forces and torques. In Figure 7 we show that the radial force PDF gets wider for denser agglomerate slurries.

\subsection{Aggregation of spherical particles in turbulence}

In the recent literature, modelling of aggregates in homogeneous deformation fields has received considerable attention (Bäbler et al., 2008; Zaccone et al., 2009; Soos et al., 2010; Higashitani et al., 2001; Becker et al., 2009; Harshe et al., 2011). The - in such cases - small size of the aggregates compared towith the fluid dynamic micro-scales allows for a Stokes flow approximation at the particle level and thus for the use of e.g., Stokesian dynamics (Brady and Bossis, 1988) to describe the interactions between the primary particles (spheres in case of Stokesian dynamics) forming aggregates, and the surrounding fluid. In such simulations, the overall deformation field that agitates the Stokesian dynamics is an input condition.

Here, we remove the assumption of aggregates being small compared to with the Kolmogorov scale. This has a few consequences. In the first place it implies that the Reynolds numbers based on aggregate size and even on primary particle size (the latter defined as $\operatorname{Re}=a^{2} \dot{\gamma} / v$ with $\dot{\gamma}$ the magnitude of the deformation rate tensor, $a$ the primary particle radius, and $v$ the kinematic viscosity of the liquid) are not necessarily (much) smaller than unity and that inertial effects (of fluid as well as of the particles) need to be resolved (and Stokesian dynamics can not be applied). It also implies that there is no clear length-scale distinction between Kolmogorov-scale flow and aggregate-scale flow anymore. Both scales overlap in an order-of-magnitude sense and directly interact. Therefore, a direct, two-way coupling between the turbulent micro-scales and the inhomogeneous fluid deformation experienced by, and generated by the moving, rotating, and continuously restructuring aggregates needs to be established.

In our simulations, HIT is generated in a cubic, fully periodic, three-dimensional domain through linear forcing (Rosales and Meneveau, 2005). In the turbulent field,

Figure 7 PDF of the normal force in sphere-sphere contact points in quadruplets. Same colour coding as Figure 6 (see online version for colours)

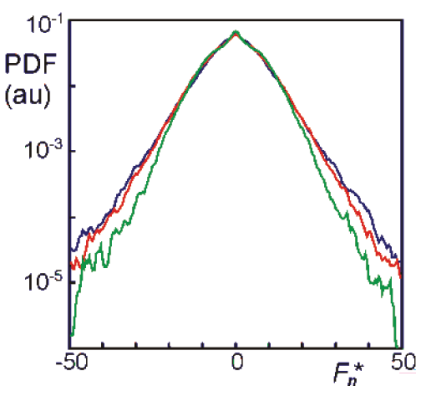

uniformly sized, spherical primary solid particles are released. The solids typically occupy $10 \%$ of the total volume.

The particles are made sticky (i.e., they have a tendency to aggregate) by means of a SqWP (see Smith et al. (1997) and the section on solid-liquid and solid-solid-coupling earlier in this paper). Next to interacting through the SqWP, the particles interact via the interstitial fluid and through hard-sphere collisions. The combination of turbulence and the SqWP produces a solid-liquid system in which bonds between primary spheres are continuously formed and broken, and an Aggregate Size Distribution (ASD) evolves naturally to a dynamically stationary state. Our main interest is how the ASD depends on turbulence properties on one side, and the interaction potential on the other.

An important question is how large the (cubic) domain needs to be to get representative results that are independent of the domain size. This is particularly relevant since our solid-liquid systems aggregate and not only the primary sphere size should be much smaller than the domain size, but also the aggregate size should be (much) smaller than the size of the domain to avoid the unphysical situation that an aggregate strongly interacts with itself through the periodic boundaries. Apart from aggregation and self-interaction between aggregates, the turbulence imposes demands on the domain size: it should have sufficient room to develop its wide spectrum of length scales to be representative for the strong turbulence in large scale process equipment. Obviously, the domain size is limited by the finite computational resources (time and memory) available. To investigate domain size effects, cubic domains with four different vertex lengths $L=128=21.3 a, L=192=32 a, L=256=42.7 a$, $L=384=64 a$ (the sphere radius a has not been varied and corresponds to 6six lattice spacings) have been considered.

Since in the larger flow domains the turbulence is allowed to generate larger structures, the time to steady state (as measured in viscous time units $a^{2} / v$ ) gets longer for larger $L$ (see Fig. 8). Three variables have been tracked in Figure 8: the ratio $\eta_{\mathrm{k}} / a$; a turbulence Reynolds number based on the volume-average root-mean-square velocity in the liquid $\operatorname{Re}_{a}=a u_{\text {rms }} / v$, and the number of attachment points per sphere $\left(n_{c}\right)$ as a metric for the level of aggregation. The stable time series (after reaching steady state) of the ratio $\eta_{k} / a$, and the good agreement between its pre-set and actual values shows that the linear forcing procedure is able to maintain a constant, desired dissipation rate; also in the presence of solids. The dissipation rate is independent of the size of the computational domain, which it should be.

The steady state level of $\mathrm{Re}_{a}$ does depend on domain size $L$; if the domain gets larger $\operatorname{Re}_{a}$ gets larger which means that the Turbulent Kinetic Energy (TKE) per unit fluid mass increases. This is dueowing to the larger, energy containing structures that fit in the larger domains. To quantify this, TKE spectra are shown in Figure 9. The spectra for different domain sizes more or less overlap for the higher wave numbers $\kappa=2 \pi / \lambda$. This is because the simulations with different domain sizes have the same dissipation rate and, therefore, develop the same small-scale turbulence. If we discard the simulations in the smallest domain $(L=128)$ 
Figure 8 Time series of key variables of aggregating spheres in homogeneous isotropic turbulence. From top to bottom: Kolmogorov length-scale over particle radius, particlesize-based Reynolds number $\operatorname{Re}_{a} \equiv u_{r m s} a / v$, number of sphere-sphere contacts per sphere. Left: frictionless collisions ( $\mu=0$ ), right: $\mu \rightarrow \infty$. The colours indicate domain size (see online version for colours)

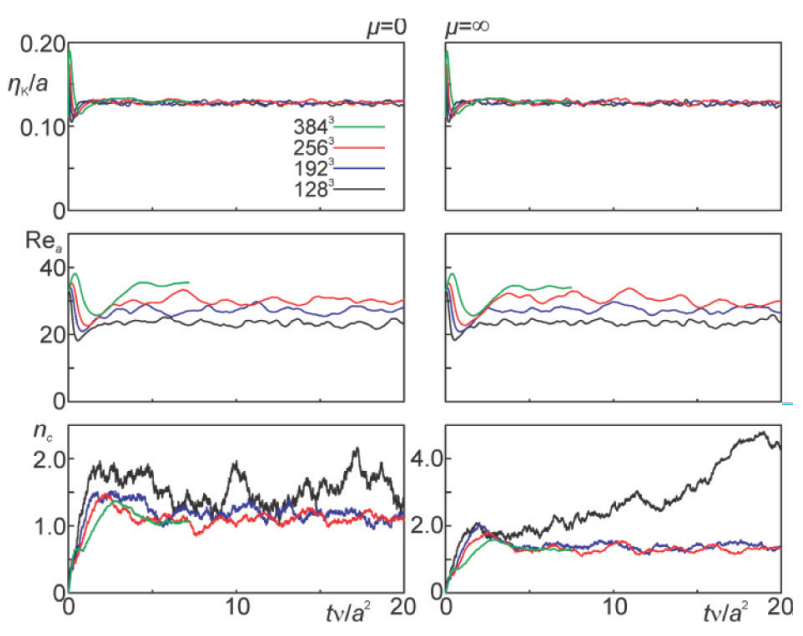

the spectra only deviate significantly for the smaller wave numbers with ${ }^{10} \log (\kappa a)<-0.5$, i.e., for flow structures with sizes larger than

$$
\frac{2 \pi a}{10^{-0.5}} \approx 20 a \text {. }
$$

As a result of this, the turbulent environment of the primary particles and also of small aggregates can be considered (statistically) similar for the simulations at different domain size, as long as $L \geq 192$. This is more likely the reason why the average number of contacts per sphere (bottom panels of Fig. 8) is approximately independent of the domain size, again as long as $L \geq 192$.

For the smallest simulations $(L=128)$, the number of contacts per sphere is clearly different, and also the spectra

Figure 9 Power spectral density of turbulent kinetic energy as a function of dimensionless wave number $\kappa a$ after steady state has been reached. The same solid-liquid systems as in Figure 8 (see online version for colours)

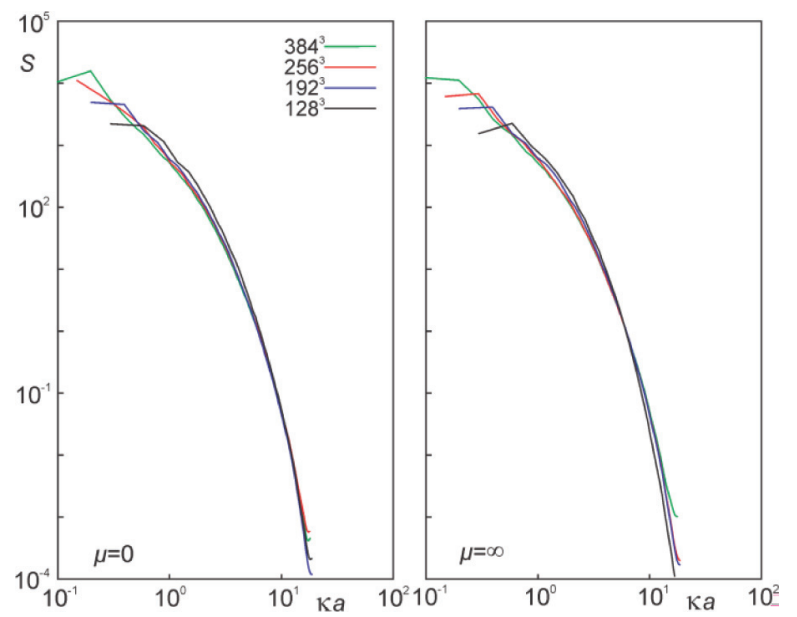

Figure 10 Single realisations of aggregates in cubic domains. Top: primary spheres coloured by the size of the aggregate they are part of (red: $n_{\text {agg }}<4$; yellow: $4 \leq n_{\text {agg }}<7$; green: $7 \leq n_{\text {agg }}<10$; blue: $n_{\text {agg }} \geq 10$ ). Top-left: $L=128$; top-right $L=384$. Cases with $\mu \rightarrow \infty$. Bottom: the four biggest aggregates for cases with $L=384$; left $\mu \rightarrow \infty$ (red: $n_{\text {agg }}=60$, yellow: $n_{\text {agg }}=150$, green $n_{\text {agg }}=65$, blue: $n_{\text {agg }}=105$; the red aggregate connects through the periodic boundaries); right $\mu=0$ (red: $n_{\mathrm{agg}}=32$, yellow: $n_{\text {agg }}=46$, green $n_{\text {agg }}=31$, blue: $n_{\text {agg }}=41$; the yellow aggregate connects through the periodic boundaries) (see online version for colours)
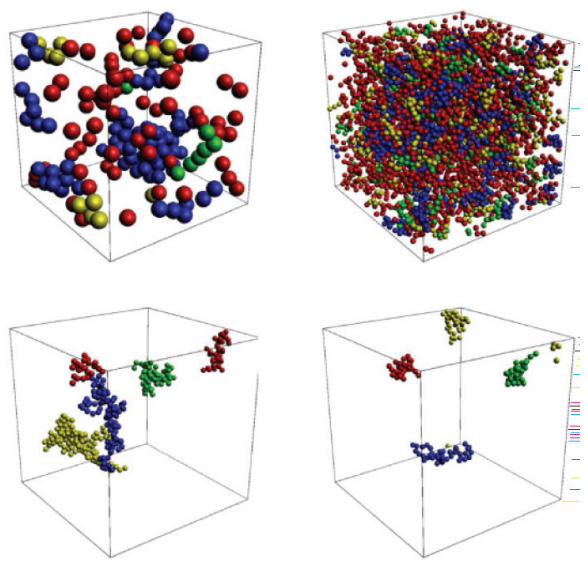

deviate over larger portions of the wave number space (Fig. 9) and we conclude that such a domain is too small. In the case with $\mu \rightarrow \infty$ a stationary state is actually not reached if $L=128$; the number of contacts per sphere keeps increasing. Closer inspection teaches that the spheres keep on aggregating and tend to form a single, big aggregate. If (for $L \geq 192$ ) steady state $n_{c}$ values are compared between $\mu=0$ and $\mu \rightarrow \infty$, friction induces higher levels of attachment of spheres and thus probably larger aggregates.

We now turn to the structure and size of the aggregates that are continuously formed and broken as a result of the turbulent flow. In Figure 10 instantaneous realisations of particle configurations are given. From the panel related to $L=128$ it may be more clear that - as argued above - this domain is too small for a domain-size-independent representation of the turbulence-aggregation interaction; larger domains are clearly needed. The bottom panels of Figure 10 show the largest aggregates at a certain (arbitrary) moment in time, suggesting larger aggregates when collisions between primary spheres are frictional. They also suggest a fairly open aggregate structure, i.e., relatively low fractal dimensions.

To make the observations in Figure 10 regarding the aggregates more quantitative and also to further investigate domain-size effects, time-averaged ASD's were determined. The ASD's presented in this paper are by aggregate mass (which is the same as by aggregate volume or by aggregate size in terms of the number of primary spheres $n_{\text {agg }}$ given the monodisperse primary spheres). To determine ASD's we took a large number of instantaneous realisations during the stationary portion $\left(t \geq\left(5\left(a^{2} / v\right)\right)\right)$ of the time series shown 
in Figure 8 (except for the case with $L=128$ and $\mu \rightarrow \infty$ that did not become steady; for this case, we also started building an ASD from $\left(t=5 \frac{a^{2}}{v}\right)$ on). The size distributions for the same cases for which we showed the time series in Figure 8 are given in Figure 11. Note that these are normalised size distributions; i.e., the area under each curve is the same. Also, note the logarithmic ordinate. For $\mu=0$ the ASD is fairly independent of $L$ as long as $L \geq 192$; the case with $L=128$ deviates strongly. The mass-averaged aggregate sizes are $\left\langle n_{\text {agg }}\right\rangle=2.60,2.07,1.97,1.88$ for $L=128,192,256,384$, respectively, which (beyond $L=128$ ) shows a weak trend towards smaller $\left\langle n_{\text {agg }}\right\rangle$ for larger domains. This may be due to the stronger turbulence (albeit at the larger scales only) for the larger domains. Also, for $\mu \rightarrow \infty$ ASD's are similar if $L \geq 192$. The average aggregate sizes are (in the order small to large domain) $4.63,2.42,2.27$, and 2.22 , i.e., slightly but significantly larger than for $\mu=0$.

Based on the basis of what was learned so far, a number of simulations were performed all having $L=256$ and $\mu \rightarrow \infty$. The settings for $\eta_{k} / a, \phi$, and $\Delta u / v$ were varied. Note that changing one dimensionless number and keeping the rest the same sometimes implies changing more than one physical parameter. For instance, a decrease in the ratio $\eta_{k} / a$ was achieved by increasing the energy dissipation rate, thus reducing the Kolmogorov length scale. At the same time the Kolmogorov velocity scale $v$ increases. In order to keep $\Delta u / v$ constant, we increase $\Delta u$ (and thus the binding energy) by the same factor as $v$ increases.

The resulting ASD's are presented in Figure 12. A striking observation is that the turbulent and aggregating solid-liquid systems can quickly get unstable. If the depth of the square well $\Delta u / v$ is increased from 0.30 to 0.35 (an increase by a factor of 1.36 in the binding energy which is proportional to $(\Delta u)^{2}$ the system slowly but consistently keeps on aggregating without reaching a steady ASD; see the lower panel of Figure 12 and its inset. If the solids volume fraction is increased from $\phi=0.08$ (base-case) to 0.16 a large aggregate consisting of the order of two thousand primary spheres is formed (the total number of primary spheres in this simulation is 2960), surrounded by a number of smaller aggregates and primary spheres; see the middle panel of Figure 12 plus inset.

Figure 11 Aggregate size distributions by mass for the cases defined in Figure 8. Comparison between frictionless (left) and frictional (right) collisions, and effects of system size (see online version for colour)

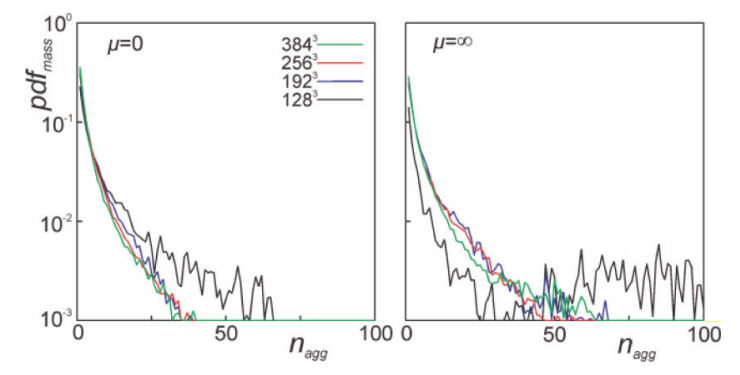

Apart from the unstable nature of some of the aggregating solid-liquid systems, the results in terms of ASD's follow expected trends: For a given primary particle size, a decrease in $\eta_{K} / a$ means a decrease in the Kolmogorov length scale as a results of an increasing energy dissipation rate. Since (unless stated otherwise) the ASD's were obtained during a stationary time window, dissipation is in equilibrium with power input, and higher dissipation implies higher power input and thus stronger turbulence. The results in the top panel of Figure 12 therefore show a shift towards smaller aggregate sizes if the power input is increased. Starting at the highest value of $\eta_{k} / a$, from one case to the next the power input increases by a factor of 4 . For $\eta_{k} / a=0.181$, $0.129,0.091$ the respective mass-average aggregate sizes $\left\langle n_{\text {agg }}\right\rangle$ are $3.99,2.27$, and 1.71 . The average aggregate size is approximately linear in $\eta_{k} / a$ in the (fairly narrow) range considered here.

It was discussed above that the denser suspensions $(f=0.16)$ we investigated got unstable. The more dilute suspension with $f=0.04$ develops much smaller aggregates compared to the $f=0.08$ base-case, largely because collisions are much less frequent in the dilute suspension (middle panel of Figure 12). Also the influence of the depth of the

Figure 12 ASD's by mass for $L=256$ domains, averaged over the time-interval (see online version for colours)

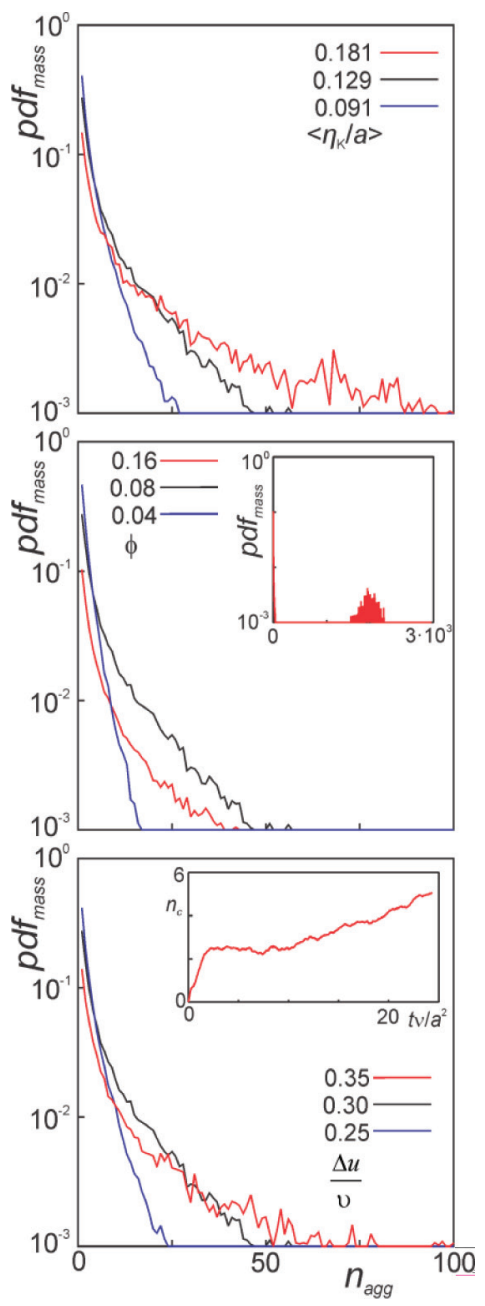


square-well follows our intuition: the shallower interaction potential leads to smaller aggregates; the deeper well to larger aggregates (bottom panel of Figure 12).

\section{Summary and perspective}

This paper presents a few case studies of mesoscopic modelling of solid-liquid flows, with underlying topics such as momentum transfer, turbulence modulation, aggregation, and flow-induced forces in and on agglomerates. Except for lubrication modelling, the simulations are direct, meaning that no (empirical) closures or empirical correlations for e.g., forces on particles enter the simulations.

From an industrial standpoint the flow systems studied are (still) very simple: monosized spherical, solid particles in Newtonian carrier fluids. The choice for monodispersed systems is not fundamental; the simulation strategy easily allows for size distributions. The extension towards non-spherical particles would be much less straightforward. Specifically in dense systems, handling collisions of non-spherical particles would get (computationally) more complicated.

The reason for the relative simplicity at the mesoscale was to keep the parameter space limited. Adding complications (at the mesoscale) strongly adds to the dimensionality of the parameter space (then size distributions, particle shape characterisation, and rheological parameters would enter). It would be useful though to add complexity to the mesoscale, the challenge being to directly mimic the interactions there and for instance see how particle shape impacts momentum transfer in dense suspensions. The price to pay for this is getting less general (i.e., work towards more and more specific applications); results only apply to the specific systems of choice; the (general) link to the macro-scale would be harder to establish.

Relating with real processes and industry in this respect is essential. Zooming in on practical systems and making choices regarding the physics to be incorporated there only pays off if it helps in solving practical problems with economical and environmental impact.

This paper is a revised and expanded version of a paper entitled 'Dense suspensions - the richness of solid-liquid interactions at the particle scale' that was presented at the CFD2011 Conference (Trondheim, Norway, June 2011).

\section{References}

Alvelius, K. (1999) 'Random forcing of three-dimensional homogeneous turbulence', Phys. Fluids, Vol. 11, pp.1880-1889.

Bäbler, M.U., Morbidelli, M. and Bałdyga, J. (2008) 'Modelling the breakup of solid aggregates in turbulent flows', J. Fluid Mech., Vol. 612, pp.261-289.

Becker, V., Schlauch, E., Behr, M. and Briesen, H. (2009) 'Restructuring of colloidal aggregates in shear flows and limitations of the free-draining approximation', J. Colloid Interface Sc., Vol. 339, pp.362-372.

Boivin, M., Simonin, O. and Squires, K.D. (1998) 'Direct numerical simulation of turbulence modulation by particles in isotropic turbulence', J. Fluid Mech., Vol. 375, pp.235-263.

Brady, J.F. and Bossis, G. (1988) 'Stokesian dynamics', Annu. Rev. Fluid Mech., Vol. 20, pp.111-157.
Chen, S. and Doolen, G.D. (1998) 'Lattice Boltzmann method for fluid flows', Annu. Rev. Fluid Mech., Vol. 30, pp. 329-364.

Delichatsios, M.A. and Probstein, R.F. (1976) 'The effect of coalescence on the average drop size in liquid-liquid dispersions', Ind. Engng. Chem. Fund., Vol. 14, pp.134-138.

Derksen, J. and Van den Akker, H.E.A. (1999) 'Large-eddy simulations on the flow driven by a Rushton turbine', AIChE J., Vol. 45, pp.209-221.

Derksen, J.J. (2008) 'Flow induced forces in sphere doublets', $J$. Fluid Mech., Vol. 608, pp.337-356.

Derksen, J.J. and Sundaresan, S. (2007) 'Direct numerical simulations of dense suspensions: wave instabilities in liquidfluidized beds', J. Fluid Mech., Vol. 587, pp.303-336.

Elghobashi, S. and Truesdell, G. (1993) 'On the two-way interaction between homogenous turbulence and dispersed solid particles. I: Turbulence modification', Phys. Fluids, Vol. 5, pp.1790-1801.

Goldstein, D., Handler, R. and Sirovich, L. (1993) 'Modeling a no-slip flow boundary with an external force field', J. Comp. Phys., Vol. 105, pp.354-366.

Harshe, Y.M., Lattuada, M. and Soos, M. (2011) 'Experimental and modeling study of breakage and restructuring of open and dense colloidal aggregates', Langmuir, Vol. 27, pp.5739-5752.

Higashitani, K., Iimura, K. and Sanda, H. (2001) 'Simulation of deformation and breakup of large aggregates in flows of viscous fluids', Chem. Engng. Sc., Vol. 56, pp.2927-2938.

Kusters, K.A. (1991) The Influence of Turbulence on Aggregation of Small Particles in Agitated Vessel, $\mathrm{PhD}$, Eindhoven University of Technology, Netherlands.

Ladd, A.J.C. (1994) 'Numerical simulations of particle suspensions via a discretized Boltzmann equation. Part 2. Numerical results', J. Fluid Mech., Vol. 271, pp.311-339.

Nguyen, N-Q. and Ladd, A.J.C. (2002) 'Lubrication corrections for lattice-Boltzmann simulations of particle suspensions', Phys. Rev. E., Vol. 66, pp.046708.

Qian, Y.H., d'Humieres, D. and Lallemand, P. (1992) 'Lattice BGK for the Navier-Stokes equations', Europhys. Lett., Vol. 17, pp.479-484.

Rosales, C. and Meneveau, C. (2005) 'Linear forcing in numerical simulations of isotropic turbulence: Physical space implementations and convergence properties', Phys. Fluids, Vol. 17, pp.095106-1-8.

Smith, S.W., Hall, C.K. and Freeman, D.B. (1997) 'Molecular dynamics for polymeric fluids using discontinuous potentials', J. Comp. Phys., Vol. 134, pp.16-30.

Somers, J.A. (1993) 'Direct simulation of fluid flow with cellular automata and the lattice-Boltzmann equation', Appl. Sci. Res., Vol. 51, pp.127-133.

Soos, M., Ehrl, L., Bäbler, M.U. and Morbidelli, M. (2010), 'Aggregate breakup in a contracting nozzle', Langmuir, Vol. 26, pp.10-18.

Soos, M., Sefcik, J. and Morbidelli, M. (2006) 'Investigation of aggregation, breakage and restructuring kinetics of colloidal dispersions in turbulent flows by population balance modeling and static light scattering', Chem. Engng. Sc., Vol. 61, pp.2349-2363.

Succi, S. (2001) The Lattice Boltzmann Equation for Fluid Dynamics and Beyond, Clarondon Press, Oxford. 
Ten Cate, A., Derksen, J.J., Portela, L.M. and Van den Akker, H.E.A. (2004) 'Fully resolved simulations of colliding spheres in forced isotropic turbulence', J. Fluid Mech., Vol. 519, pp.233-271.

Ten Cate, A., Nieuwstad, C.H., Derksen, J.J. and Van den Akker, H.E.A. (2002) 'PIV experiments and lattice-Boltzmann simulations on a single sphere settling under gravity', Phys. Fluids, Vol. 14, pp.4012-4025.

Ten Cate, A., Van Vliet, E., Derksen, J.J. and Van den Akker, H.E.A. (2006) 'Application of spectral forcing in lattice-Boltzmann simulations of homogeneous turbulence', Computers and Fluids, Vol. 35, pp.1239-1251.

Yamamoto, Y., Potthoff, M., Tanaka, T., Kajishima, T. and Tsuji, Y. (2001) 'Large-eddy simulation of turbulent gas-particle flow in a vertical channel: effect of considering inter-particle collisions', J. Fluid Mech., Vol. 442, pp.303-334.

Zaccone, A., Soos, M., Lattuda, M., Wu, H., Bäbler, M.U. and Morbidelli, M. (2009) 'Breakup of dense colloidal aggregates under hydrodynamic stresses', Phys. Rev. E, Vol. 79, pp.061401. 